\title{
Outcomes of Modestly Hypofractionated Radiation for Lung Tumors: Pre- and Mid-Treatment Positron Emission Tomography-Computed Tomography Metrics as Prognostic Factors
}

\author{
Jeremy P. Harris, ${ }^{1}$ Christine N. Chang-Halpenny, ${ }^{1,2}$ Peter G. Maxim, ${ }^{1,3}$ \\ Andrew Quon, ${ }^{3,4}$ Edward E. Graves, ${ }^{1,3}$ Maximilian Diehn, ${ }^{1,3,5}$ Billy W. Loo, Jr ${ }^{1,3}$
}

\begin{abstract}
Many patients with lung tumors have tumors too large for stereotactic ablative radiotherapy and comorbidities precluding concurrent chemotherapy. We report the outcomes of 29 patients treated with hypofractionated radiotherapy (RT) to 60 to 66 Gy in 3-Gy fractions. We also report an exploratory analysis of the prognostic value of the pre- and mid-RT positron emission tomography-computed tomography.

Introduction: Modestly hypofractionated radiation therapy (HypoRT; 60-66 Gy in 3-Gy fractions) allows patients with locally advanced thoracic tumors and poor performance status to complete treatment within a shorter period without concurrent chemotherapy. We evaluated the outcomes and imaging prognostic factors of HypoRT. Materials and Methods: We retrospectively reviewed the data from all patients with primary and metastatic intrathoracic tumors treated with HypoRT from 2006 to 2012. We analyzed the survival and toxicity outcomes, including overall survival (OS), progression-free survival (PFS), local recurrence (LR), and distant metastasis. We also evaluated the following tumor metrics in an exploratory analysis: gross tumor volume (GTV), maximum standardized uptake value $\left(\mathrm{SUV}_{\mathrm{Max}}\right)$, and metabolic tumor volume using a threshold of $\geq 50 \%$ of the SUV $\mathrm{Sax}\left(\mathrm{MTV}_{50 \%}\right)$ or the maximum gradient of fluorine18 fluorodeoxyglucose uptake (MTV Edge). We assessed the association of these metrics and their changes from before to mid-RT using positron emission tomography-computed tomography (PET-CT) with OS and PFS. Results: We identified 29 patients, all with pre-RT and 20 with mid-RT PET-CT scans. The median follow-up period was 15 months. The 2-year overall and non-small-cell lung cancer-only rate for OS, PFS, and LR, was $59 \%$ and $59 \%, 52 \%$ and $41 \%$, and $27 \%$ and $32 \%$, respectively. No grade $\geq 3$ toxicities developed. The median decrease in GTV, SUV ${ }_{\text {Max }}$, and MTV Edge $_{\text {Eas } 11 \%, 24 \% \text {, and } 18 \% \text {, respectively. Inferior OS was associated with a larger pre-RT MTV }}$ Edge $(P=.005)$ and pre-RT $\mathrm{MTV}_{50 \%}(P=.007)$. Inferior PFS was associated with a larger mid-RT SUV $\operatorname{Max}(P=.003)$. Conclusion: These findings add to the growing body of data demonstrating promising outcomes and limited toxicity with HypoRT. The pre- and mid-RT PET-CT metrics could be useful for prognostic stratification in future clinical trials.
\end{abstract}

Clinical Lung Cancer, Vol. 16, No. 6, 475-85 @ 2015 Elsevier Inc. All rights reserved.

Keywords: Hypofractionation, Lung neoplasms, PET scan, Prognostication, Radiotherapy

J.P.H. and C.C.-H. contributed equally to this work as first authors.

${ }^{1}$ Department of Radiation Oncology, Stanford University School of Medicine, Stanford, CA

${ }^{2}$ Department of Radiation Oncology, Kaiser Permanente Los Angeles Medical Center, Los Angeles, CA

${ }^{3}$ Stanford Cancer Institute, Stanford University School of Medicine, Stanford, CA

${ }^{4}$ Division of Nuclear Medicine, Department of Radiology, Stanford University School of Medicine, Stanford, CA
${ }^{5}$ Institute for Stem Cell Biology and Regenerative Medicine, Stanford University School of Medicine, Stanford, CA

Submitted: Nov 4, 2014; Revised: Jan 20, 2015; Accepted: Jan 23, 2015; Epub: Jan 31, 2015

Addresses for correspondence: Billy W. Loo, Jr, MD, PhD and Maximilian Diehn, MD, $\mathrm{PhD}$, Department of Radiation Oncology and Stanford Cancer Institute, Stanford University School of Medicine, 875 Blake Wilbur Drive, MC 5847, Stanford, CA 94305-5847 E-mail contact: BWLoo@Stanford.edu and Diehn@Stanford.edu 


\section{Introduction}

Modestly hypofractionated radiation therapy (HypoRT) allows for treatment completion within a shorter period and could be advantageous for patients with locally advanced lung tumors and a poor performance status or an inability to tolerate concurrent chemotherapy. HypoRT results in fewer daily treatments and a reduced cost compared with conventionally fractionated RT (CFRT) or combined chemotherapy and RT (chemoRT). Although small tumors can be treated effectively and safely with stereotactic ablative radiotherapy (SABR), a more fractionated approach is generally considered necessary for safe treatment of more advanced disease. Studies have shown effective treatment is attained with HypoRT for both early-stage non-small-cell lung cancer (NSCLC) and late-stage NSCLC and, with more accelerated courses, for oligometastatic lung cancer. ${ }^{1-10}$ One concern is the potential for increased toxicity from HypoRT. However, the reported toxicities have generally been found to be acceptable compared with CFRT with appropriate patient selection and conformal radiation techniques. Although HypoRT can be effective, currently, limited prognostic factors are available to guide treatment and predict the outcomes.

Fluorine-18 fluorodeoxyglucose (FDG) positron emission tomography (PET) combined with computed tomography (PET-CT) is an important tool for staging primary lung cancer and could potentially be used as a prognostic indicator of disease control and patient survival. Determining the metrics for tumor response could be used to tailor and potentially adjust treatment. Previous studies have demonstrated the usefulness of the CT-determined gross tumor volume (GTV) and metabolic tumor volume (MTV) for predicting the outcomes for NSCLC after both CFRT and SABR. ${ }^{11-16}$ Additionally, analysis of the mid-RT PET-CT findings of different radiation fractionations and doses for lung tumors has shown that a decrease in FDG uptake is associated with improved outcomes. ${ }^{17-20}$ To our knowledge, no other studies have been conducted that examined the benefit of mid-RT PET-CT with regard to MTV or as a prognosticator for patients undergoing HypoRT.

We sought to demonstrate the prognostic usefulness of pre- and mid-RT PET-CT metrics and to share our institutional experience of patients with lung cancer treated with a modestly hypofractionated course (60-66 Gy in 3-Gy fractions) of RT.

\section{Materials and Methods Patient Population}

After approval by our institutional review board, we reviewed the medical records of all patients with an intrathoracic tumor treated with a modestly hypofractionated course of RT (60-66 Gy in 3-Gy fractions) using either 3-dimensional conformal (3D-CRT) or intensity-modulated RT (IMRT) at the Stanford Cancer Institute from January 2006 to January 2012. Intrathoracic tumors were those located in the lung cavity or thoracic lymph nodes. The patients included those with both primary or metastatic tumors. During this period, HypoRT was selected for patients with locally advanced or limited metastatic disease. This included patients with stage I lung cancer with a tumor volume too large for SABR and patients with nodal disease and a relatively compact overall planning target volume (PTV), who were considered poor candidates for chemoRT owing to a poor performance status. The pathologic specimens were reviewed at our institution. All patients underwent a pre-RT PET-CT scan within 1 month before RT. No exclusions were made for age, race, gender, or performance status.

\section{Evaluation and Treatment}

The pre-RT evaluation included history, physical examination, and diagnostic PET-CT. A total of 29 patients were identified. RT planning and delivery was by either IMRT or 3D-CRT: 19 patients were treated with conventional IMRT, 9 with volumetric modulated arc therapy, and 1 with 3D-CRT. Of the 29 patients, 28 were considered inoperable, and 1 patient underwent upper and lower wedge resections for a stage T2aN0M0 bronchogenic carcinoma followed by RT for positive surgical margins. This patient was not a candidate for lobectomy because of major chronic obstructive pulmonary disease comorbidity. For patients with stage I to III disease, treatment was with the intent to cure. For those with stage IV cancer, all known disease sites were targeted with definitive intent, except for 1 patient, who was treated with palliative intent. That patient had several metastatic lesions from a primary rectal adenocarcinoma and had received treatment to a mediastinal nodal mass causing new-onset left recurrent laryngeal nerve palsy. Six patients had undergone chemotherapy before RT, and 3 patients had undergone chemotherapy after RT. No patient was treated concurrently.

The GTV was contoured on axial slices of the planning CT scans, with a pulmonary window setting for pulmonary parenchymal lesions and a mediastinal window setting for mediastinal lesions, with the aid of fused PET-CT. No explicit expansion was made for microscopic extension to form the clinical target volume to minimize the normal tissue toxicity in a relatively frail patient population using this hypofractionated regimen. However, the multibeam arrangements used resulted in a relatively isotropic dose gradient such that the microscopic control doses (45-50 Gy in accelerated fractionation) typically extended $\geq 0.5$ to $1 \mathrm{~cm}$ beyond the high-dose PTV. Respiratory motion was managed using a motion-inclusive internal target volume (ITV), determined using 4-dimensional CT. The ITV was expanded by a $0.5-\mathrm{cm}$ circumferential margin to define the PTV. The treatment planning goals included $\geq 95 \%$ PTV coverage with the prescription dose, with a minimum dose of $\geq 90 \%$ (ideally $\geq 95 \%$ ) and maximum dose of $\leq 115 \%$ (ideally $\leq 110 \%$ ) of the prescription dose. All treatments were delivered using 6-MV photons. Image guidance was performed using daily pretreatment orthogonal kilovoltage imaging and at least weekly (typically twice weekly) cone-beam CT.

The dose regimens ranged from 60 to $66 \mathrm{~Gy}$, delivered in 3-Gy fractions. Because ours was not a prospective trial, the normal tissue constraints were at the discretion of the treating physicians. However, given the accelerated schedule, they were generally more conservative than the ideal constraints we have typically used in our clinic for conventionally fractionated thoracic IMRT to $\geq 60 \mathrm{~Gy}$ (eg, percentage of lung volume receiving $20 \mathrm{~Gy} \leq 30 \%$, mean lung dose $\leq 18 \mathrm{~Gy}$, mean esophageal dose $\leq 25 \mathrm{~Gy}$, maximum spinal cord dose $\leq 45 \mathrm{~Gy}$, percentage of heart volume receiving 30 Gy $\leq 50 \%$ ). In most cases, the doses achieved were substantially lower than these constraints, reflecting the high conformity of the plans. However, in individual cases, structures overlapping the PTV could receive doses exceeding them within a small volume of tissue. 
The treatment parameters, including the normal tissue doses as delivered, are described in Table 1.

\section{PET-CT Imaging}

At our institution, patients with thoracic tumors treated with HypoRT often undergo PET-CT as part of the initial staging, as described previously, and they frequently undergo PET-CT during their radiation course as a part of adaptive replanning and to rule out interval metastases that would result in premature RT completion. ${ }^{12,15,21,22}$ PET-CT was performed after a 4- to 8-hour fast, followed by an injection of 10 to $18 \mathrm{mCi}$ of FDG, with imaging 45 to 60 minutes later on a GE Discovery ST PET-CT scanner (GE Medical Systems, Milwaukee, WI). Helical CT imaging was performed for attenuation correction. The blood glucose levels were all between 80 and $160 \mathrm{mg} / \mathrm{dL}$ at injection.

\section{PET-CT Analysis}

PET-CT analyses were performed similarly to those in our previous investigations. ${ }^{15,22}$ The maximum standardized uptake value $\left(\mathrm{SUV}_{\mathrm{Max}}\right)$ within the GTV was determined for both the pre- and mid-RT scans. The volume within the GTV with an SUV $\geq 50 \%$ of the $\mathrm{SUV}_{\mathrm{Max}}$ defined the metabolic tumor volume at the $50 \%$ level $\left(\mathrm{MTV}_{50 \%}\right)$. The mid-RT MTV $50 \%$ was not analyzed, because the mid-RT SUV Max $_{\text {was }}$ generally decreased compared with the

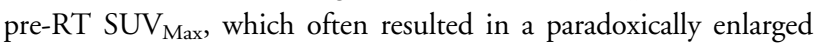
mid-RT $\mathrm{MTV}_{50 \%}$. We also computed the volume according to the steepest gradient of FDG uptake, the so-called PET-Edge calculation $\left(\mathrm{MTV}_{\mathrm{Edge}}\right){ }^{23} \mathrm{~A}$ mid-RT GTV was calculated using a deformed contour of the original GTV. The planning CT scan overlaid the mid-RT CT with a rigid box focused on the tumor and lungs. The planning CT volume was translated into a corresponding mid-RT CT volume using a deformable registration algorithm focused on the predefined rigid box space. The GTV contour followed the same translation to become a GTV of the mid-RT CT scan. The mid-RT GTVs were manually adjusted by us and confirmed by a radiation oncologist. Likewise, all other tumor volumes were evaluated by 1 of us and confirmed by a radiation oncologist. Lesions were considered PET negative (volume, $0 \mathrm{~cm}^{3}$ ) when the background SUV was indistinguishable from that within the GTV on the mid-RT PET scan. We used the MIM Maestro software suite, version 5.1 (MIM Software, Inc., Cleveland, OH), for PET-CT analysis and deformable image registration.

\section{Outcome Analysis and Statistical Analysis}

Changes in tumor metrics were assessed using Wilcoxon signedrank tests. The first follow-up assessment was typically 3 months after treatment completion and included a PET-CT or CT scan. The patients then underwent follow-up assessments at approximately 3-month intervals with clinic visits and PET-CT or CT. Toxicity was assessed using the Common Terminology Criteria for Adverse Events, version 3.0. The primary endpoints of overall survival (OS) and progression-free survival (PFS) were determined for each patient. PFS was defined as freedom from any disease progression (local, regional, or distant) or death from any cause. Disease progression was determined from radiologic findings that were backdated to the first abnormality. The Kaplan-Meier method was used to generate survival curves. Local recurrence (LR) and distant metastasis (DM) were estimated using competing risk analyses, where death was a competing risk. The differences in outcomes for the tumor metrics were estimated using Cox proportional hazards models. Hazard ratios represented a $10-\mathrm{cm}^{3}$ increase in tumor volume or a 1-unit increase in $S_{U V}$ Max. The prognostic factors were evaluated using univariate Cox proportional hazards models, and included Karnofsky performance status (KPS), American Joint Committee on Cancer stage IV disease, age, and gender. Inspection of log-log plots and a time-interaction variable was used to test the proportional hazards assumption.

We used the Bonferroni-Holm method to adjust for multiple comparisons of tumor metrics. Seven tumor metrics were compared; thus, the highest significance was set at $P<.0071$. All statistical tests were 2-tailed. Statistical analysis was performed using the Statistical Analysis Systems, version 9.3 (SAS Institute, Inc., Cary, NC).

\section{Results}

\section{Patient and Treatment Outcomes}

Our review identified 29 patients who met the inclusion criteria. One patient died before the 3-month follow-up examination, and the rest were evaluated during the follow-up period. The patient characteristics are summarized in Table 1.

The median follow-up period for all patients was 15 months (range, 4-50 months) and for the surviving patients was 10 months (range, 4-50 months). Of the 29 patients, 12 (41\%) developed local progression after RT, with a median interval of 10 months (range, 5-32 months). The 1- and 2-year LR rate was 23\% (95\% confidence interval [CI] 9\%-41\%) and $27 \%$ (95\% CI $11 \%-45 \%$ ), respectively. Of the 29 patients, $16(55 \%)$ developed DM, with a median interval of 7 months (range, 3-41 months). The 1- and 2-year DM rate was 46\% (95\% CI 26\%-65\%) and 51\% (95\% CI $29 \%-68 \%$ ), respectively. At the last follow-up examination, 8 patients $(28 \%)$ were living. The 1 - and 2-year OS rate was $85 \%$ (95\% CI 64\%-94\%) and 59\% (95\% CI 37\%-76\%), respectively. The 1- and 2-year PFS rate was $60 \%$ (95\% CI 36\%-77\%) and 52\% (95\% CI 28\%-72\%). Figure 1 shows the outcomes for the entire and NSCLC cohorts.

\section{Toxicity}

A total of 3 patients developed grade 2 esophagitis, 2 developed grade 2 pneumonitis, and 1 developed grade 2 dermatitis. No grade $\geq 3$ complications developed. The toxicity outcomes are also summarized in Table 1.

\section{PET-CT Metrics}

All patients underwent a pre-RT PET-CT scan. The median interval from PET-CT to RT was 13 days (range, 7-28 days). Of the 29 patients, 20 underwent a mid-RT PET-CT scan, which was performed a median of 16 days (range, 10-27 days) from the start of RT. The main analysis was performed on the entire cohort, with a subgroup analysis on the patients with NSCLC (NSCLC cohort).

The median GTV, SUV $\mathrm{Sax}_{\text {Max }}, \mathrm{MTV}_{50 \%}$, and $\mathrm{MTV}_{\text {Edge }}$ was 37.3 $\mathrm{cm}^{3}, 13.1,7.8 \mathrm{~cm}^{3}$, and $16.4 \mathrm{~cm}^{3}$. One patient had a GTV of 569 $\mathrm{cm}^{3}, 4.9$ standard deviations greater than the mean, and was excluded from the remaining analysis to meet the linearity assumption for Cox regression models and so that the associations 
Table 1 Patient, Tumor, Treatment Characteristics, and Toxicity Events

\begin{tabular}{|c|c|c|}
\hline Characteristic & $\begin{array}{l}\text { Entire Cohort } \\
\qquad(n=29)\end{array}$ & $\begin{array}{c}\text { NSCLC } \\
(n=19)\end{array}$ \\
\hline \multicolumn{3}{|l|}{ Patient characteristic } \\
\hline \multicolumn{3}{|l|}{ Gender (n) } \\
\hline Male & $20(69)$ & $12(63)$ \\
\hline Female & $9(31)$ & $7(37)$ \\
\hline \multicolumn{3}{|l|}{ Age (years) } \\
\hline Median (IQR) & $73(60-83)$ & $81(71-85)$ \\
\hline Mean \pm SD & $72 \pm 13$ & $76 \pm 12$ \\
\hline \multicolumn{3}{|c|}{ Stage at treatment $(\mathrm{n})$} \\
\hline 1 & $4(14)$ & $4(21)$ \\
\hline$\|$ & $5(17)$ & $5(26)$ \\
\hline NO & & 3 \\
\hline N1 & & 2 \\
\hline III & $6(21)$ & $5(26)$ \\
\hline NO (T4NO) & & 3 \\
\hline N1 (T4N1) & & 1 \\
\hline N2 (T1N2) & & 1 \\
\hline IV & $14(48)$ & $5(26)$ \\
\hline NO & & 3 \\
\hline $\mathrm{N} 1$ & & 1 \\
\hline N2 & & 1 \\
\hline \multicolumn{3}{|l|}{ Histologic type } \\
\hline Adenocarcinoma & $8(28)$ & $8(42)$ \\
\hline SCC & $6(21)$ & $6(32)$ \\
\hline Other NSCLC & $5(17)$ & $5(26)$ \\
\hline Thymic SCC & $1(3)$ & $0(0)$ \\
\hline Metastasis & $9(31)$ & $0(0)$ \\
\hline \multicolumn{3}{|l|}{ Location } \\
\hline Upper lobe & $15(52)$ & $10(53)$ \\
\hline Middle lobe & $6(21)$ & $4(21)$ \\
\hline Lower lobe & $8(28)$ & $5(26)$ \\
\hline \multicolumn{3}{|l|}{ Other } \\
\hline \multicolumn{3}{|c|}{ Performance status, KPS } \\
\hline Median (IQR) & $90(70-90)$ & $80(70-90)$ \\
\hline Mean \pm SD & $81 \pm 12$ & 79 (12) \\
\hline \multicolumn{3}{|c|}{ Tumor and treatment characteristics } \\
\hline \multicolumn{3}{|l|}{ Radiation dose (Gy) } \\
\hline 60 & $6(21)$ & $3(16)$ \\
\hline 63 & $1(3)$ & $0(0)$ \\
\hline 66 & $22(76)$ & $16(84)$ \\
\hline \multicolumn{3}{|l|}{ Mean lung dose (Gy) } \\
\hline Median & 8.5 & 8.1 \\
\hline Minimum & 1.4 & 1.4 \\
\hline IQR & $6.1-10.7$ & $6.1-11.1$ \\
\hline Maximum & 20.6 & 15.3 \\
\hline \multicolumn{3}{|l|}{ Lung $\mathrm{V}_{20}(\%)$} \\
\hline Median & 12 & 12 \\
\hline Minimum & 1 & 1 \\
\hline IQR & $9-18$ & $10-18$ \\
\hline Maximum & 42 & 23 \\
\hline
\end{tabular}

Table 1 Continued

\begin{tabular}{|c|c|c|}
\hline Characteristic & $\begin{array}{l}\text { Entire Cohort } \\
\qquad(\mathrm{n}=29)\end{array}$ & $\begin{array}{c}\text { NSCLC } \\
(n=19)\end{array}$ \\
\hline \multicolumn{3}{|l|}{ Mean esophageal dose (Gy) } \\
\hline Median & 8.1 & 7.7 \\
\hline Minimum & 0.2 & 0.2 \\
\hline IQR & $4.1-10.4$ & 4.3-9.9 \\
\hline Maximum & 39.5 & 18.9 \\
\hline \multicolumn{3}{|l|}{ Esophagus $V_{30}(\%)$} \\
\hline Median & 2 & 2 \\
\hline Minimum & 0 & 0 \\
\hline IQR & $0-11$ & $0-9$ \\
\hline Maximum & 59 & 32 \\
\hline \multicolumn{3}{|l|}{ Esophageal $V_{60}(\%)$} \\
\hline Median & 0 & 0 \\
\hline Minimum & 0 & 0 \\
\hline IQR & $0-1$ & $0-0$ \\
\hline Maximum & 31 & 4 \\
\hline \multicolumn{3}{|l|}{$\begin{array}{l}\text { Maximum spinal canal } \\
\text { dose (Gy) }\end{array}$} \\
\hline Median & 26.6 & 23.1 \\
\hline Minimum & 0.8 & 1.0 \\
\hline IQR & $16.5-33.7$ & $16.7-32.6$ \\
\hline Maximum & 61.7 & 45.9 \\
\hline Resection & $1(3)$ & $1(5)$ \\
\hline \multicolumn{3}{|l|}{ Chemotherapy before RT } \\
\hline No & $23(79)$ & $16(84)$ \\
\hline Carboplatin/pemetrexed & $1(3)$ & $1(5)$ \\
\hline $\begin{array}{l}\text { Carboplatin/pemetrexed/ } \\
\text { bevacizumab }\end{array}$ & $1(3)$ & 1 (5) \\
\hline Carboplatin/gemcitabine & $1(3)$ & $1(5)$ \\
\hline $\begin{array}{l}\text { Carboplatin/cetuximab/ } \\
\text { docetaxel }\end{array}$ & 1 (3) & $0(0)$ \\
\hline Cetuximab/irinotecan & $1(3)$ & $0(0)$ \\
\hline Sorafenib & $1(3)$ & $0(0)$ \\
\hline \multicolumn{3}{|l|}{ Chemotherapy after RT } \\
\hline No & $26(90)$ & 18 (95) \\
\hline Pemetrexed & $1(3)$ & $1(5)$ \\
\hline Carboplatin/gemcitabine & $1(3)$ & $0(0)$ \\
\hline $\begin{array}{l}\text { Cisplatin/Adriamycin/vincristine/ } \\
\text { cyclophosphamide }\end{array}$ & 1 (3) & $0(0)$ \\
\hline \multicolumn{3}{|l|}{ Toxicity events ${ }^{\mathrm{a}}$} \\
\hline Esophagitis, grade 2 & $3(10)$ & $2(11)$ \\
\hline Pneumonitis, grade 2 & $2(7)$ & $2(11)$ \\
\hline Dermatitis, grade 2 & $1(3)$ & $1(5)$ \\
\hline
\end{tabular}

Data in parentheses are percentages, unless otherwise noted.

Abbreviations: IQR $=$ interquartile range; $\mathrm{KPS}=$ Karnofsky performance status; NSCLC $=$ non-small-cell lung cancer; RT = radiotherapy; SCC = squamous cell carcinoma; $V_{20}, V_{30}$, $\mathrm{V}_{60}=$ percentage of volume receiving 20,30 , or 60 Gy, respectively.

${ }^{\mathrm{a}}$ No grade $>3$ toxicity events occurred.

between the tumor metrics and outcome would not be controlled or masked by this outlier. The new median GTV, SUV $\mathrm{Max}, \mathrm{MTV}_{50 \%}$, and $\mathrm{MTV}_{\text {Edge }}$ was $37.0 \mathrm{~cm}^{3}, 13.9,7.3 \mathrm{~cm}^{3}$, and $16.4 \mathrm{~cm}^{3}$, respectively. The median decrease in GTV, $\mathrm{SUV}_{\mathrm{Max}}$, and $\mathrm{MTV}_{\mathrm{Edge}}$ 


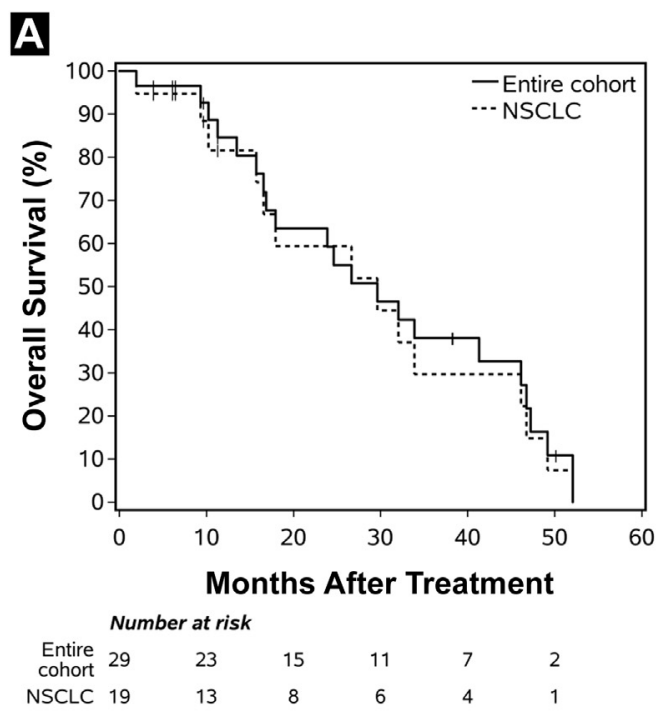

C

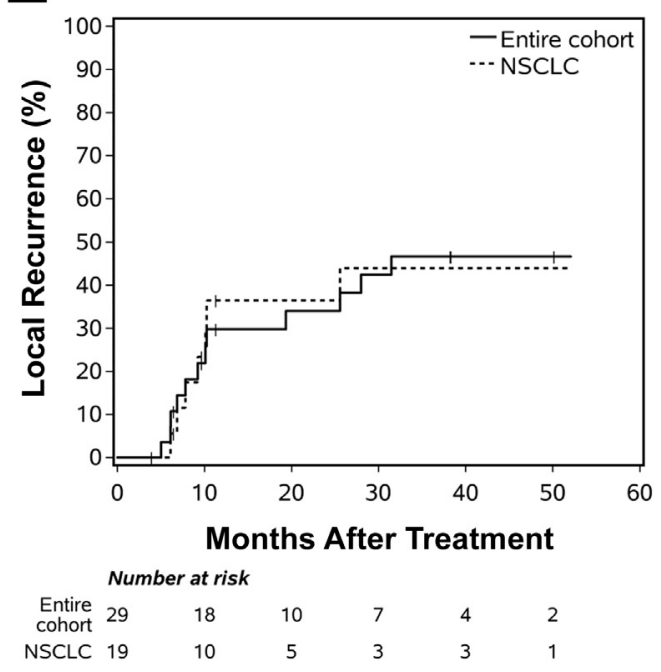

B

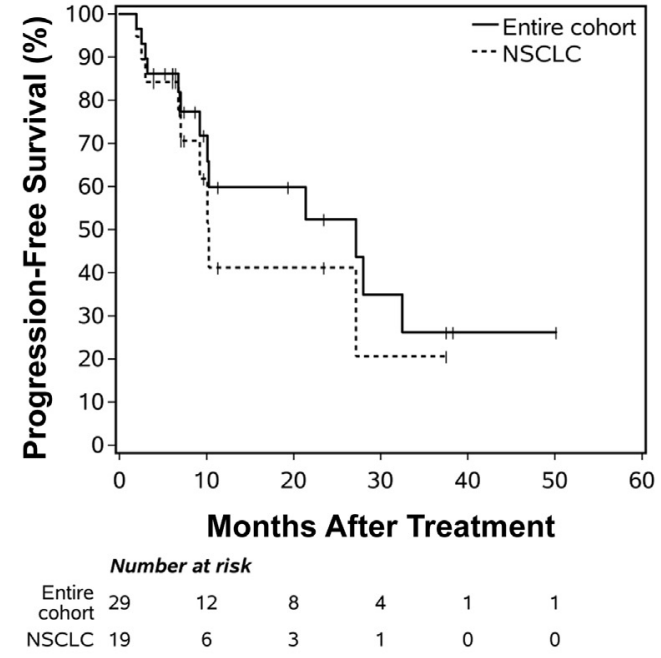

D

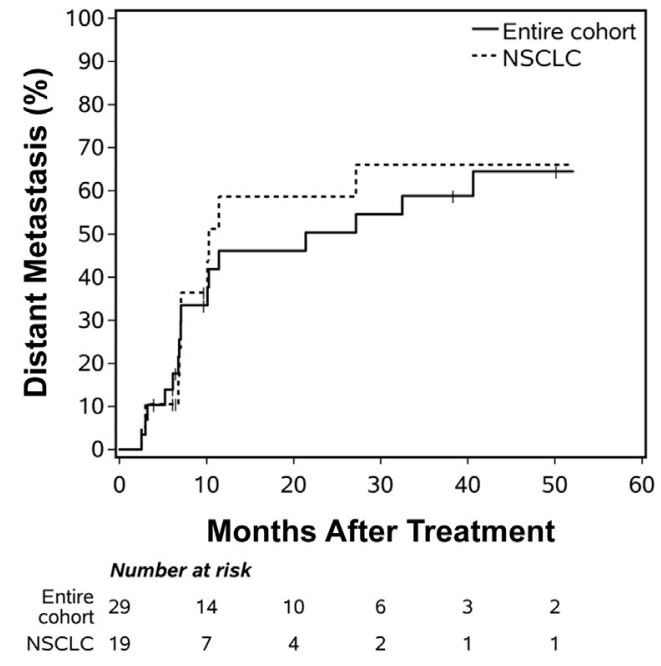

Abbreviation: NSCLC $=$ non-small-cell lung cancer.

was $11 \%, 24 \%$, and $18 \%$, respectively. All decreases in these tumor imaging metrics were significant (Figure 2).

\section{Clinical and Imaging Associations With Outcomes}

The association between the covariates and OS and PFS was estimated using unadjusted Cox regression analysis. Improved OS was associated with increasing KPS $(P=.0001)$. Improved PFS was associated with increasing KPS $(P=.03)$, younger age $(P=.04)$, and female gender $(P=.007)$.

The association between each tumor metric and outcome was estimated using unadjusted Cox regression analysis. After Bonferroni-Holm correction, an inferior OS was associated with a larger pre-RT $\operatorname{MTV}_{\text {Edge }}(P=.005)$ and a larger pre-RT $\mathrm{MTV}_{50 \%}$
$(P=.007)$. Inferior PFS was associated with a larger mid-RT $\operatorname{SUV}_{\text {Max }}(P=.003)$. Figure 3 shows the results for all models. Subgroup analysis of the NSCLC cohort revealed that these associations were present, but not significant, after statistical correction ( $P=.023, P=.025$, and $P=.0091$, respectively). The proportional hazards assumption was met for all models. The results of the Kaplan-Meier analysis for the metrics of interest, stratified by the median values, is shown in Figure 4. Log-rank tests revealed that the groups stratified by pre-RT MTV $\mathrm{Edge}_{\text {and mid-RT }}$ SUV $\mathrm{Max}_{\mathrm{Max}}$ were significantly different; however, the stratified pre-RT MTV $\mathrm{M}_{50 \%}$ groups only showed a trend toward significance $(P=.1)$.

Figure 5 shows the PET-CT scans from 2 patients with a different mid-RT SUV Max $_{\text {and }}$ correspondingly different progression 

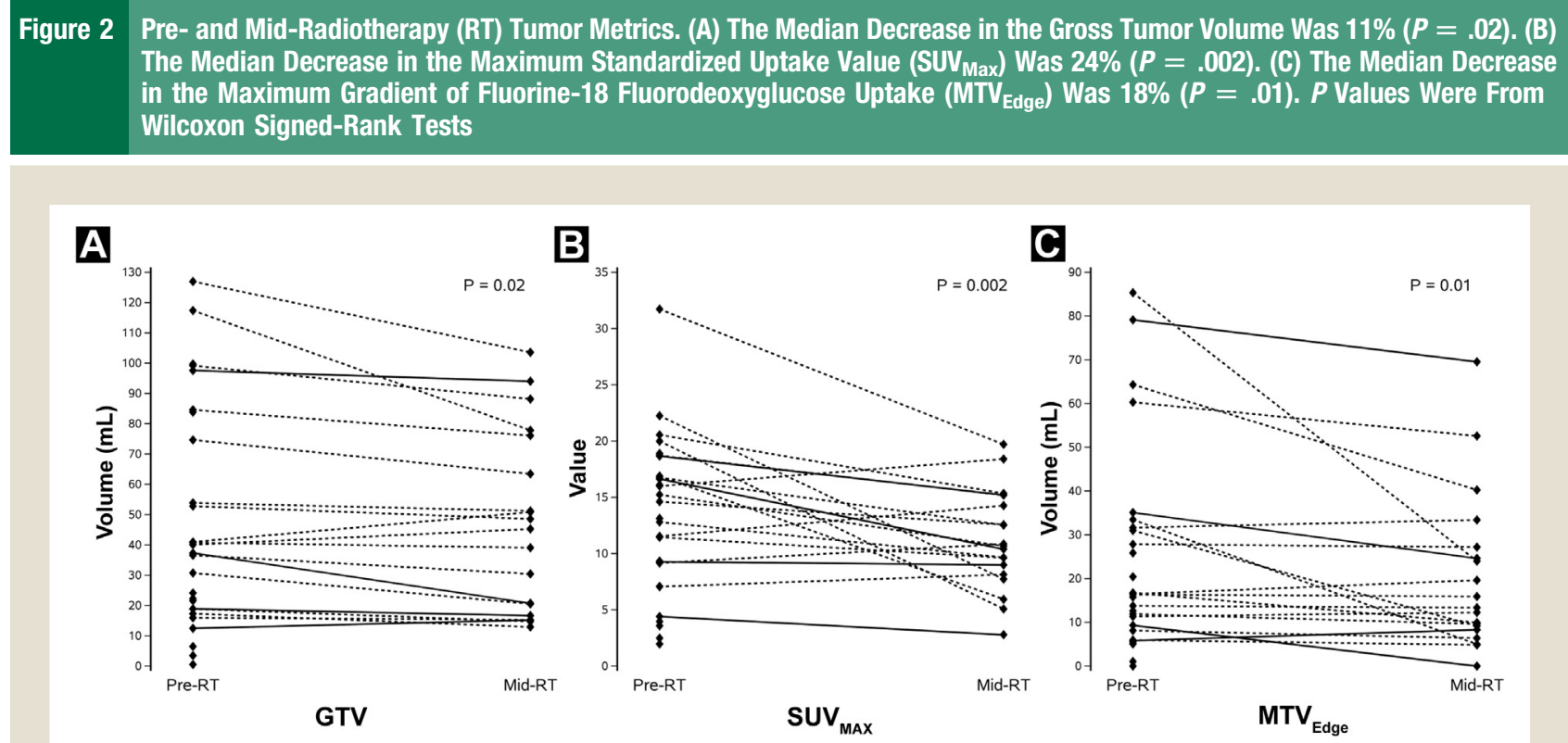

outcomes. Both patients had initially presented with stage IB, T2N0M0, squamous cell carcinoma, and both patients were treated with 66 Gy in 3-Gy fractions. The first patient (Figure 5, row 1) had had a decrease in the SUV $V_{\text {Max }}$ and MTV Edge on the mid-RT PET-CT scan and had not developed LR by 24 months. The second patient (Figure 5, row 2) had presented with a slightly smaller GTV but had had a slight increase in the $S U V_{\text {Max }}$ and $M T V_{\text {Edge }}$ on the mid-RT PET-CT scan, and had developed LR by 9 months after RT.

\section{Discussion}

We sought to share our institutional experience of patients with lung tumors treated with HypoRT and to demonstrate the prognostic usefulness of pre- and mid-RT PET-CT. The pre-RT PET-CT metrics and the change in SUV measured from the midRT PET scan have been shown to be useful tools for predicting the outcomes after RT for patients with lung cancer. ${ }^{11-20}$ We focused our study on a cohort of patients with primary and metastatic lung tumors who had undergone modestly HypoRT using 60 to $66 \mathrm{~Gy}$ in 3-Gy fractions. We found that the pre-RT MTV Edge was the most robust prognosticator of OS compared with the SUV $_{\text {Max }}, M_{50 \%}$, and GTV and that the mid-RT SUV $V_{\text {Max }}$ had the most robust association with PFS.

HypoRT has potential utility for patients with either a tumor burden too great to treat safely with aggressively HypoRT (ie, SABR), despite a technically early or an oligometastatic stage, or an inability to tolerate combined modality chemoRT (typically using CFRT) for more advanced-stage disease because of comorbidities or a poor performance status. Patient suitability for systemic therapy was determined by a multidisciplinary evaluation that included medical oncologists. We have typically considered nodal involvement or stage T3 and most stage T2B primary tumors to be too extensive to treat with SABR, because of the excessive doses to the normal tissues. However, the tumor burden can be too excessive even for HypoRT if it is not possible to achieve normal tissue doses lower than the maximum constraints we typically use for CFRT (eg, bulky or bilateral nodal disease and/or multilobar involvement). Thus, HypoRT was reserved for patients with intermediate-volume disease who were not appropriate candidates for concurrent chemotherapy. These typically frailer patients also tended to benefit logistically from a relatively short treatment course. Logistical considerations, in addition to the normal tissue doses, were factored into deciding the number of fractions, within a relatively narrow range (20-22 fractions) for individual patients.

With regard to early-stage NSCLC, an increasing number of studies have reported on the same or similar HypoRT regimens. HypoRT is often used for early-stage NSCLC when the patient is not a surgical candidate and SABR is not an option. Oh et $\mathrm{al}^{24}$ reported on a cohort of 60 patients with stage T1N0 to T3N0 NSCLC who were not SABR candidates. The treatment dose was 60 Gy in 3-Gy fractions, with a 2-year OS rate of 59.6\% and LR rate of $42.1 \% .^{24}$ Bonfili et $\mathrm{al}^{5}$ reported that for 36 patients with stage I to II NSCLC treated with $60 \mathrm{~Gy}$ in 3-Gy fractions, the 2-year OS, PFS, and LR was 55.6\%, 38.9\%, and $36.1 \%$, respectively. Yung et $\mathrm{al}^{9}$ reported on 60 patients with stage T1N0 to T2N0 NSCLC treated with a median of 60 Gy in 3-Gy fractions and found a 2 -year OS rate of $61 \%$ and LR rate of $8 \%$. The National Cancer Institute of Canada Clinical Trials Group BR25 was a phase II study of 80 patients with stage T1N0 to T3N0 NSCLC treated with modestly HypoRT to a dose of 60 Gy in 4-Gy fractions. ${ }^{25}$ The study reported a 2-year OS, PFS, and LR rate of $69 \%, 64 \%$, and $13 \%$, respectively. ${ }^{25}$ Similarly, Soliman et $\mathrm{al}^{26}$ reported on 118 patients treated with 48 to $60 \mathrm{~Gy}$ in 4-Gy fractions and found a 2-year OS rate of $51.0 \%$ and LR rate of $23.8 \%$. Finally, Bogart et $\mathrm{al}^{27}$ reported on the results from a phase I study of patients with stage I NSCLC treated with a variety of accelerated courses (70 Gy in fractions of 2.41-4.11 Gy), with a median OS of 38.5 months and median PFS of 28.6 months. 


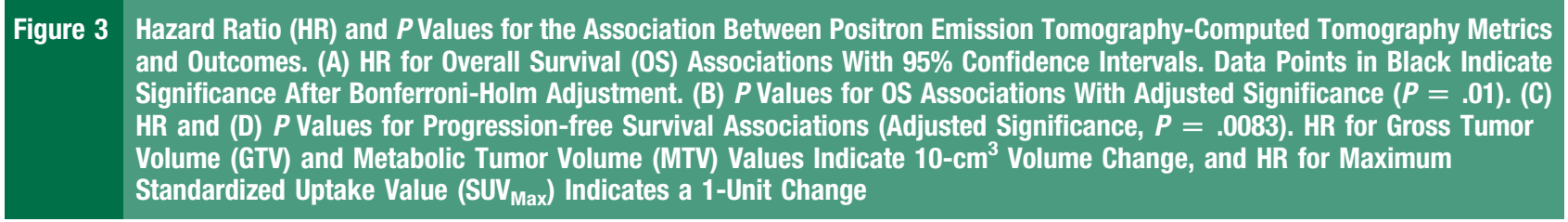

A

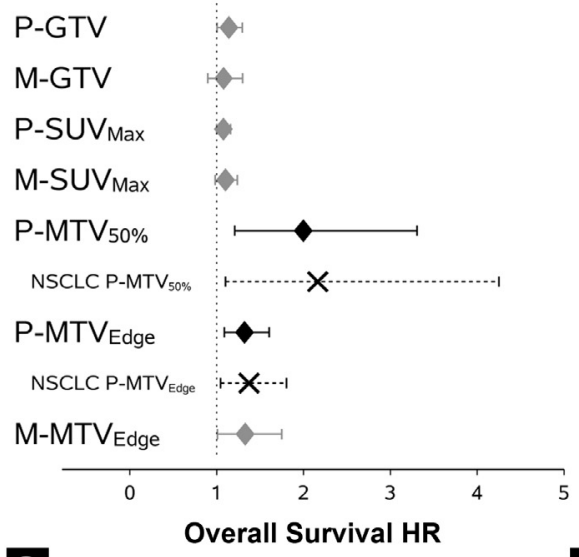

C

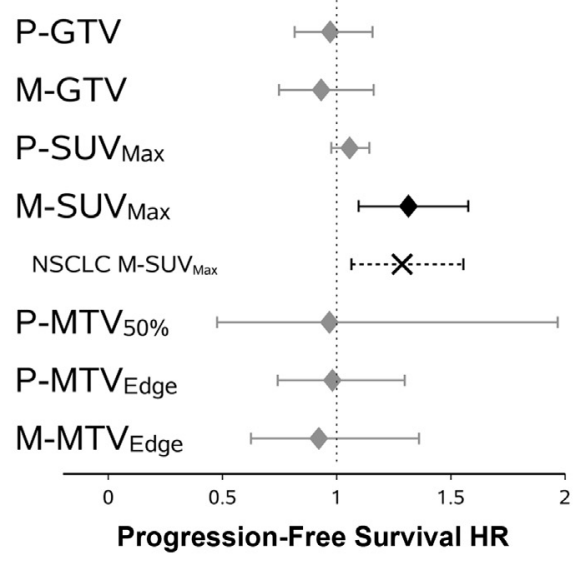

B

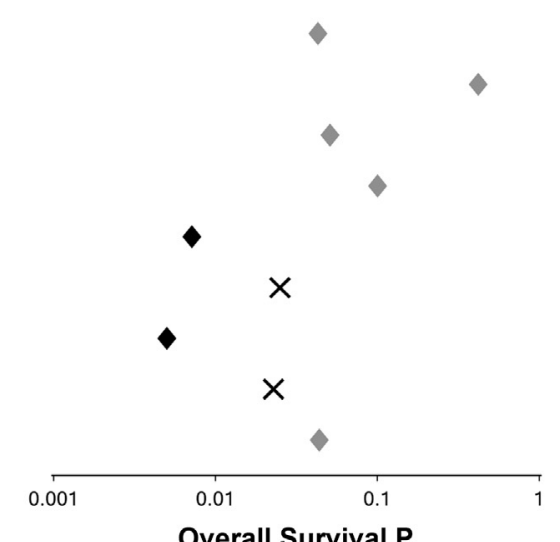

D

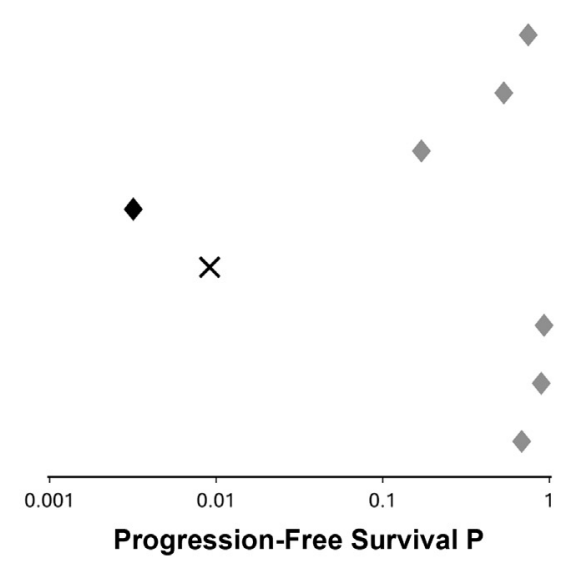

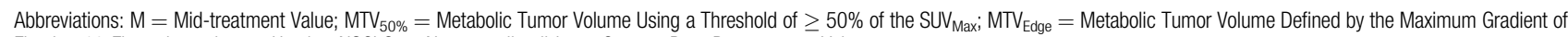
Fluorine-18 Fluorodeoxyglucose Uptake; NSCLC $=$ Non-small-cell Lung Cancer; $\mathrm{P}=$ Pretreatment Value.

Thus, for early-stage NSCLC, HypoRT can result in an estimated 2-year OS, PFS, and LR rate of $51 \%$ to $69 \%, 39 \%$ to $65 \%$, and $8 \%$ to $42 \%$, respectively. We also found that HypoRT resulted in satisfactory outcomes for NSCLC. Our estimated 2-year OS was 59\% (95\% CI 30\%-80\%), PFS 41\% (95\% CI $14 \%-67 \%$ ), and LR $32 \%$ (95\% CI 10\%-56\%). However, $52 \%$ of our NSCLC cohort had advanced-stage disease (stage III-IV). Reports from advanced stage NSCLC treated with HypoRT have tended to have inferior outcomes. Osti et al reported on 30 stage III-IV NSCLC patients treated with 60 Gy in 3 Gy per fraction, and found a 2 -year OS of $38.1 \%$, PFS $36 \%$, and LR $58.9 \% .{ }^{10}$ However, similar to the mixed population in this study, Thirion et al reported on 25 patients with stage I-III NSCLC treated with dose escalation to $72 \mathrm{~Gy}$ in 3 Gy per fraction, with a 1 -year OS of $68 \%$ and LR $28 \%{ }^{3}$ Our 1-year OS was $82 \%$
(95\% CI 53\%-94\%) and LR 32\% (95\% CI $10 \%-56 \%)$. A number of prospective trials have evaluated modestly HypoRT concurrent with chemotherapy for locally advanced NSCLC, including the European Organization for Research and Treatment of Cancer 08972-22973, Korean Radiation Oncology Group 0301, and SOCCAR trials. ${ }^{28-30}$ The radiation dose intensity in these trials was slightly lower than the HypoRT regimen in the present study, likely because concurrent chemotherapy was used in those trials, and the 2-year OS rate was $34 \%$ to $56 \%$.

Overall, our outcomes with HypoRT were similar to those for early-stage NSCLC, although our cohort presented with more advanced-stage disease. We included advanced-stage NSCLC in the present study because for this subgroup of patients a benefit exists for HypoRT compared with CFRT in accelerating treatment and reducing patient visits. Increasing the dose per fraction 


\section{Outcomes of HypoRT for Lung Tumors}

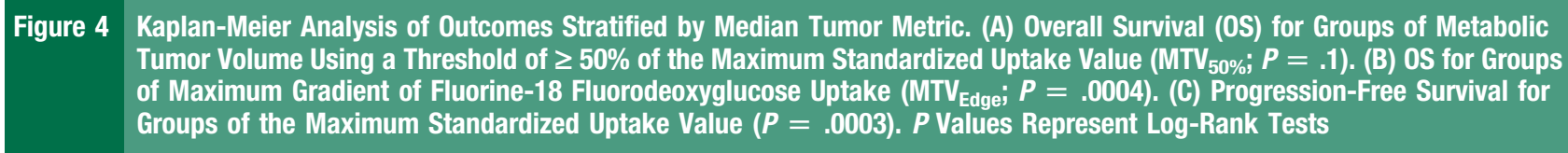

A

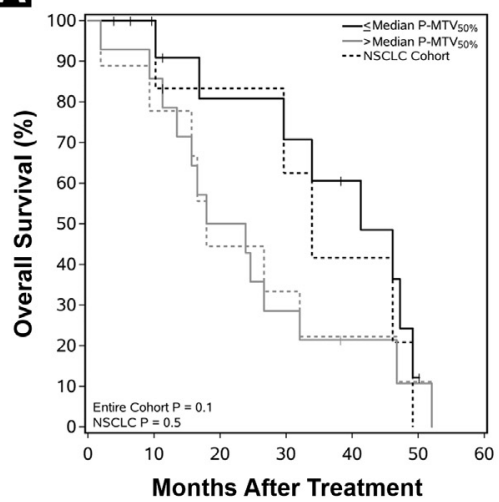

B

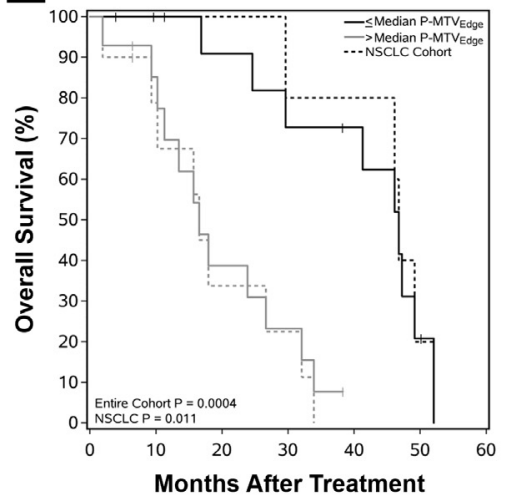

C

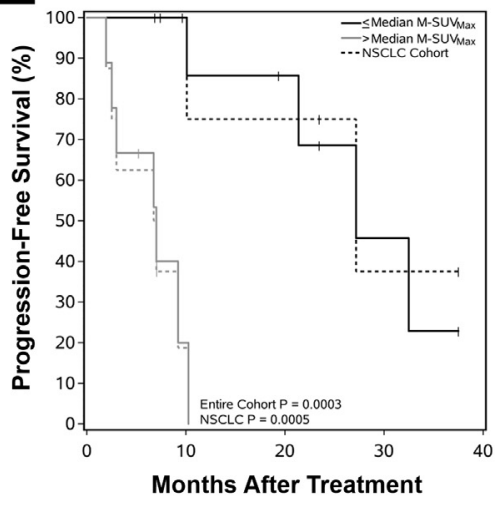

Abbreviations: $\mathrm{M}=$ Mid-treatment value; NSCLC $=$ Non-small-cell Lung Cancer; $\mathrm{P}=$ Pretreatment value.

Figure 5 Pre-Radiotherapy (RT), Mid-RT, and Follow-Up Positron Emission Tomography-Computed Tomography Scans From 2 Patients Who Both Initially Presented With Stage IB (T2NOMO) Squamous Cell Carcinoma and Were Treated With 66 Gy in $3-$ Gy Fractions But Had Different Mid-RT Maximum Standardized Uptake Values (SUV Max $_{\text {) and Correspondingly Different }}$ Progression Outcomes. Row 1: Patient With Right Upper Lobe Tumor With Pre-RT Gross Tumor Volume (GTV) of $74.7 \mathrm{~cm}^{3}$, SUV $_{\text {Max }}$ of 20.0, and Metabolic Tumor Volume Maximum Gradient of Fluorine-18 Fluorodeoxyglucose Uptake (MTV $64.3 \mathrm{~cm}^{3}$, and Left Lung Scarring From Previous Chemoradiation for Left Upper Lobe Stage IIIB Non-small-cell Lung Cancer.

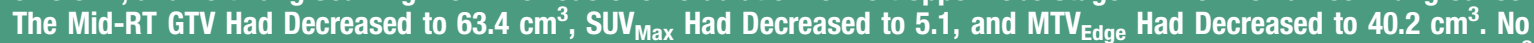
Evidence of Disease (NED) Was Seen by 24 Months. Row 2: Patient With Left Lower Lobe Tumor With Pre-RT GTV of $52.8 \mathrm{~cm}^{3}$,

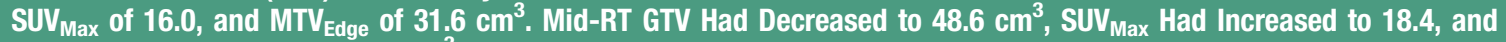
MTV $_{\text {Edge }}$ Had Increased to $33.4 \mathrm{~cm}^{3}$. This Patient Had Developed Local Recurrence (LR) at 9 Months

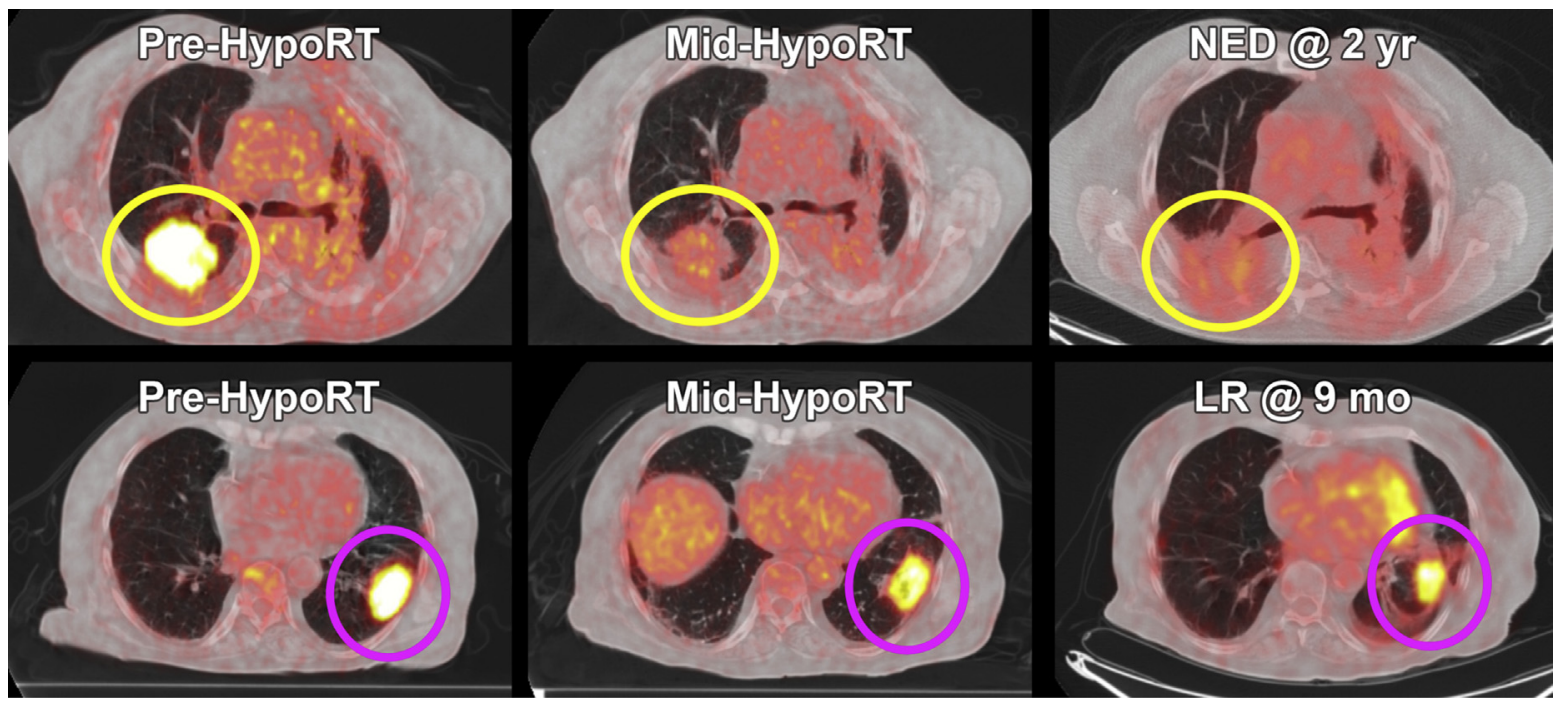

Abbreviations: Pre-HypoRT = Scan Taken Before Hypofractionated Radiotherapy; Mid-HypoRT = Scan Taken During Hypofractionated Radiotherapy. 
also raises concerns of toxicity. However, similar to previous studies demonstrating the safety of HypoRT, our study had no grade $\geq 3$ toxicity. ${ }^{6}$ Most of the patients in our cohort received doses to critical normal organs that were substantially lower than the constraints used in CFRT regimens (Table 1), reflecting the selection of patients with relatively compact tumor volumes even in locally advanced stages. In summary, our study adds to the growing body of data demonstrating good outcomes and limited toxicity with HypoRT.

PET has been a valuable tool for evaluating the NSCLC response and detecting disease. ${ }^{16,31}$ Recent studies have indicated that the usefulness of PET extends to prognostication. ${ }^{32-35}$ MacManus et $\mathrm{al}^{36}$ reported that the responses measured by PET were superior to those from CT for post-RT scans for predicting OS. Our results have similarly indicated that the PET metrics $\left(\mathrm{SUV}_{\mathrm{Max}}, \mathrm{MTV}_{50 \%}\right.$, and $\mathrm{MTV}_{\mathrm{Edge}}$ ) were superior to those from CT (GTV) for predicting OS and PFS.

Multiple studies have demonstrated the importance of pre-RT PET-CT for predicting outcomes. GTV, as both a continuous and a binary variable, has been associated with OS, cause-specific survival, and LR. 5,11,13,24,26,37 Previously, with a smaller subset of patients, we showed a correlation between PFS and the mid-RT $\mathrm{SUV}_{\mathrm{Max}}{ }^{17}$; however, no other studies have focused on mid-RT PET-CT scans for patients undergoing HypoRT.

Other studies have been directed toward evaluating sequential PET-CT scans for CFRT or SABR. van Elmpt et $\mathrm{al}^{19}$ reported the pre- and mid-RT PET-CT metrics for patients with stage II to IV NSCLC treated with larger fractionation regimens (1.8 Gy per fraction or 1.5 Gy per fraction, followed by dose escalation with 2 Gy per fraction). They found a correlation between a survival of $>2$ years and a decrease in the mean SUV and SUV $\mathrm{Sax}_{\text {ax }}$ but not the primary tumor volume, GTV, or $\mathrm{MTV}_{50 \%} \cdot{ }^{19}$ No significant correlation was found between OS and the pre- and mid-RT meanSUV or $S U V_{\text {Max }}$. Usmanij et al ${ }^{20}$ reported the pre- and mid-RT PET metrics for patients with stage III NSCLC treated with concurrent chemoRT at a dose of 2 Gy per fraction to $66 \mathrm{~Gy}$. That study found a correlation between PFS and the pre-RT total lesion glycolysis (TLG; the product of $\mathrm{MTV}_{50 \%}$ and the average SUV) and the change in TLG from the pre- to mid-RT PET, but not the $\mathrm{SUV}_{\mathrm{Max}}$. Kong et al ${ }^{18}$ similarly found that for patients with stage I to III NSCLC, the normalized mid-RT SUV $\mathrm{Max}_{\text {ax }}$ correlated with OS and PFS.

We performed an exploratory analysis on the change in the PET-CT metrics from pre- to mid-RT and found that a larger percentage of a decrease in MTV $\mathrm{Edge}_{\text {dge }}$ from pre- to mid-RT PETCT was associated with improved $\operatorname{LR}(P=.03)$. However, we did not find a correlation with the $\mathrm{SUV}_{\mathrm{Max}}$. The difference between the studies could have been related to the amount of total radiation delivered by the time of mid-RT PET-CT. The mean interval from the start of RT to the mid-RT PET-CT scan in the study by van Elmpt et $\mathrm{al}^{19}$ was 8.5 days (range, 6-13 days). However, in our study, the mean interval was 16.4 days (median, 16 days; range, 10-27 days). Additionally, our study showed a correlation between the mid-RT metrics and outcomes, which has similarly been demonstrated for CFRT for advanced-stage NSCLC. ${ }^{38,39}$ However, the results from the treatment response prediction studies have been mixed. ${ }^{40-43}$
Our experience has demonstrated that the pre- and mid-RT tumor metrics are both strongly associated with OS and PFS. Both are likely important for predicting patient outcomes and potentially for risk stratification or adjusting treatment with dose escalation. One small study of 14 patients was able to use the response during RT, measured by tumor size from PET and CT, to successfully perform dose escalation on 4 patients. ${ }^{44}$ However, our study is the first to report a significant decrease in GTV, SUV Max $_{\text {, }}$ and $\mathrm{MTV}_{\text {Edge }}$ for patients undergoing HypoRT. The current NRG Oncology cooperative group trial, Radiation Therapy Oncology Group 1106, is designed to determine the usefulness of adaptive replanning using the mid-RT PET-CT findings for patients with stage III NSCLC undergoing concurrent chemoRT with carboplatin/paclitaxel followed by consolidation chemotherapy. If this concept is found to be promising, our results suggest that a similar adaptive replanning technique could be applied to a prospective study for patients undergoing HypoRT.

The strengths of the present study included the variety of PET-CT metrics collected and tumors analyzed. As imaging technology advances, determining the best clinical predictor of patient outcomes will remain important. This is highlighted by our experience with very large tumors with central necrosis, which are measured accurately using the GTV and MTV Edge but are grossly underestimated using the $\mathrm{MTV}_{50 \%}$. The weaknesses of our study were similar to those of other single-institution retrospective studies. HypoRT is often only used for high-risk patients who are not surgical or SABR candidates, and the results were based on a small cohort, making it difficult to generalize to specific populations. In addition, our study population was heterogeneous with respect to tumor type and stage, use and type of chemotherapy, and timing and acquisition of the PET-CT scans. For a more thorough analysis of outcomes for more specific patient populations, future prospective trials are needed. Our subgroup analysis of the NSCLC cohort demonstrated similar trends with respect to the prognostic value of the imaging metrics as for the overall cohort, but it had too few patients to demonstrate statistical significance.

\section{Conclusion}

We have shared our institutional experience that HypoRT is a safe and effective method for treating primary and metastatic lung tumors in selected patients. We also performed a hypothesisgenerating study that demonstrated the usefulness of pre- and mid-RT PET-CT. The PET metrics $\left(\mathrm{MTV}_{\text {Edge }}, \mathrm{MTV}_{50 \%}\right.$, and $\left.S_{U} V_{M a x}\right)$ predicted OS and PFS better than did the CT metrics (pre- and mid-RT GTV). If validated in future randomized studies, these imaging techniques could help risk stratify patients and modify treatments for patients undergoing HypoRT for primary and metastatic lung cancer.

\section{Clinical Practice Points}

- Some nonsurgical patients with early-stage lung cancer are ineligible for SABR because of an excessive tumor size.

- Some with more advanced lung cancer are ineligible for concurrent chemotherapy because of comorbidities.

- Modestly HypoRT (60-66 Gy in 3-Gy fractions) allows for tolerable treatment within a shorter period. 
- For early-stage NSCLC, HypoRT can result in an estimated 2 -year OS of $51 \%$ to $69 \%$, PFS of $39 \%$ to $65 \%$, and LR of $8 \%$ to $42 \%$.

- Our estimated 2-year OS was 59\%, PFS 41\%, and LR 32\%; however, $52 \%$ of our NSCLC cohort had stage III to IV disease.

- Furthermore, we found no grade $\geq 3$ toxicities with the HypoRT regimen.

- We also performed an exploratory analysis of the prognostic value of pre- and mid-RT PET-CT.

- We evaluated the GTV, SUV

- Recent studies have shown the usefulness of pre- and mid-RT PET-CT metrics for prognosticating outcomes with CFRT courses.

- Our study has demonstrated similar correlations for HypoRT.

- We found that inferior OS was associated with a larger pre-RT $\operatorname{MTV}_{\text {Edge }}(P=.005)$ and pre-RT $\operatorname{MTV}_{50 \%}(P=.007)$ and inferior PFS with a larger mid-RT $\operatorname{SUV}_{\mathrm{Max}}(P=.003)$.

- HypoRT offers certain patients a shorter RT course.

- Future prospective trials could lead to using objective pre- and mid-RT PET-CT data to both risk-stratify patients and adjust the radiation dose during treatment.

\section{Acknowledgments}

J.P.H. and C.N.C. were supported by the Stanford Medical Scholars Fellowship Program.

\section{Disclosure}

B.W.L., M.D., P.G.M., and E.E.G. have received research support from Varian Medical Systems. B.W.L. and P.G.M. have received research support from RaySearch Laboratories and speaking honoraria from Varian Medical Systems.

\section{References}

1. Simpson JR, Bauer M, Wasserman TH, et al. Large fraction irradiation with or without misonidazole in advanced non-oat cell carcinoma of the lung: a phase III randomized trial of the RTOG. Radiation Therapy Oncology Group. Int J Radiat Oncol Biol Phys 1987; 13:861-7.

2. Slotman BJ, Antonisse IE, Njo KH. Limited field irradiation in early stage (T1-2N0) non-small cell lung cancer. Radiother Oncol 1996; 41:41-4.

3. Thirion P, Holmberg O, Collins CD, et al. Escalated dose for non-small-cell lung cancer with accelerated hypofractionated three-dimensional conformal radiation therapy. Radiother Oncol 2004; 71:163-6.

4. Kepka L, Tyc-Szczepaniak D, Bujko K. Dose-per-fraction escalation of accelerated hypofractionated three-dimensional conformal radiotherapy in locally advanced non-small cell lung cancer. J Thorac Oncol 2009; 4:853-61.

5. Bonfili P, Di Staso M, Gravina GL, et al. Hypofractionated radical radiotherapy in elderly patients with medically inoperable stage I-II non-small-cell lung cancer. Lung Cancer 2010; 67:81-5.

6. Carruthers R, O'Rourke N, Mohammed N, et al. Toxicity of hypofractionated accelerated radiotherapy concurrent with chemotherapy for non-small cell carcinoma of the lung. Clin Oncol 2011; 23:561-2.

7. Zhu ZF, Fan M, Wu KL, et al. A phase II trial of accelerated hypofractionated three-dimensional conformal radiation therapy in locally advanced non-small cell lung cancer. Radiother Oncol 2011; 98:304-8.

8. Chang HJ, Ko HL, Lee CY, et al. Hypofractionated radiotherapy for primary or secondary oligometastatic lung cancer using tomotherapy. Radiat Oncol 2012; $7: 222$.

9. Yung T, Giuliani ME, Le LW, et al. Outcomes of accelerated hypofractionated radiotherapy in stage I non-small-cell lung cancer. Curr Oncol 2012; 19:e264-9.

10. Osti MF, Agolli L, Valeriani M, et al. Image guided hypofractionated 3-dimensional radiation therapy in patients with inoperable advanced stage non-small cell lung cancer. Int J Radiat Oncol Biol Phys 2013; 85:e157-63.

11. Bradley JD, Ieumwananonthachai N, Purdy JA, et al. Gross tumor volume, critical prognostic factor in patients treated with three-dimensional conformal radiation therapy for non-small-cell lung carcinoma. Int J Radiat Oncol Biol Phys 2002; 52:49-57.
12. Lee P, Weerasuriya DK, Lavori PW, et al. Metabolic tumor burden predicts for disease progression and death in lung cancer. Int J Radiat Oncol Biol Phys 2007; 69: 328-33.

13. Werner-Wasik M, Swann RS, Bradley J, et al. Increasing tumor volume is predictive of poor overall and progression-free survival: secondary analysis of the Radiation Therapy Oncology Group 93-11 phase I-II radiation dose-escalation study in patients with inoperable non-small-cell lung cancer. Int J Radiat Oncol Biol Phys 2008; 70:385-90.

14. Kozak MM, Murphy JD, Schipper ML, et al. Tumor volume as a potential imaging-based risk-stratification factor in trimodality therapy for locally advanced non-small cell lung cancer. J Thorac Oncol 2011; 6:920-6.

15. Abelson JA, Murphy JD, Trakul N, et al. Metabolic imaging metrics correlate with survival in early stage lung cancer treated with stereotactic ablative radiotherapy. Lung Cancer 2012; 78:219-24.

16. Lee P, Bazan JG, Lavori PW, et al. Metabolic tumor volume is an independent prognostic factor in patients treated definitively for non-small-cell lung cancer. Clin Lung Cancer 2012; 13:52-8.

17. Chang CN, Olson M, Weerasuriya DK, et al. Mid-radiation FDG-PET is a prognostic factor for disease progression in patients with non-small cell lung cancer. Presented at the 94th Radiological Society of North America 2008 Scientific Assembly and Annual Meeting, Chicago, IL, 2008:RO32-02.

18. Kong F, Ten Haken RK, Gross M, et al. FDG-PET/CT during radiation therapy to predict survival and guide individualized adaptive dose escalation in patients with non-small-cell lung cancer. Int J Radiat Oncol Biol Phys 2009; 75:S35-6.

19. van Elmpt W, Ollers M, Dingemans AM, et al. Response assessment using 18F-FDG PET early in the course of radiotherapy correlates with survival in advanced-stage non-small cell lung cancer. J Nucl Med 2012; 53:1514-20.

20. Usmanij EA, de Geus-Oei LF, Troost EG, et al. 18F-FDG PET early response evaluation of locally advanced non-small cell lung cancer treated with concomitant chemoradiotherapy. J Nucl Med 2013; 54:1528-34.

21. Schellenberg D, Quon A, Minn AY, et al. ${ }^{18}$ Fluorodeoxyglucose PET is prognostic of progression-free and overall survival in locally advanced pancreas cancer treated with stereotactic radiotherapy. Int J Radiat Oncol Biol Phys 2010; 77:1420-5.

22. Murphy JD, La TH, Chu K, et al. Postradiation metabolic tumor volume predicts outcome in head-and-neck cancer. Int J Radiat Oncol Biol Phys 2011; $80: 514-21$.

23. Wanet M, Lee JA, Weynand B, et al. Gradient-based delineation of the primary GTV on FDG-PET in non-small cell lung cancer: a comparison with thresholdbased approaches, CT and surgical specimens. Radiother Oncol 2011; 98:117-25.

24. Oh D, Ahn YC, Kim B, et al. Hypofractionated three-dimensional conformal radiation therapy alone for centrally located cT1-3N0 non-small-cell lung cancer. I Thorac Oncol 2013; 8:624-9.

25. Cheung P, Faria S, Ahmed S, et al. Phase II study of accelerated hypofractionated three-dimensional conformal radiotherapy for stage T1-3 N0 M0 non-small cell lung cancer: NCIC CTG BR.25. I Natl Cancer Inst 2014:106.

26. Soliman H, Cheung P, Yeung L, et al. Accelerated hypofractionated radiotherapy for early-stage non-small-cell lung cancer: long-term results. Int J Radiat Oncol Biol Phys 2011; 79:459-65.

27. Bogart JA, Hodgson L, Seagren SL, et al. Phase I study of accelerated conformal radiotherapy for stage I non-small-cell lung cancer in patients with pulmonary dysfunction: CALGB 39904. J Clin Oncol 2010; 28:202-6.

28. Belderbos J, Uitterhoeve L, van Zandwijk N, et al. Randomised trial of sequential versus concurrent chemo-radiotherapy in patients with inoperable non-small cell lung cancer (EORTC 08972-22973). Eur J Cancer 2007; 43:114-21.

29. Cho KH, Ahn SJ, Pyo HR, et al. A phase II study of synchronous threedimensional conformal boost to the gross tumor volume for patients with unresectable stage III non-small-cell lung cancer: results of Korean Radiation Oncology Group 0301 study. Int J Radiat Oncol Biol Phys 2009; 74:1397-404.

30. Maguire J, Khan I, McMenemin R, et al. SOCCAR: a randomised phase II trial comparing sequential versus concurrent chemotherapy and radical hypofractionated radiotherapy in patients with inoperable stage III non-small cell lung cancer and good performance status. Eur J Cancer 2014; 50:2939-49.

31. MacManus MP, Hicks RJ, Matthews JP, et al. Metabolic (FDG-PET) response after radical radiotherapy/chemoradiotherapy for non-small cell lung cancer correlates with patterns of failure. Lung Cancer 2005; 49:95-108.

32. Higashi K, Ueda Y, Arisaka Y, et al. 18F-FDG uptake as a biologic prognostic factor for recurrence in patients with surgically resected non-small cell lung cancer. J Nucl Med 2002; 43:39-45.

33. Pottgen C, Levegrun S, Theegarten D, et al. Value of 18F-fluoro-2-deoxy-Dglucose-positron emission tomography/computed tomography in non-small-cell lung cancer for prediction of pathologic response and times to relapse after neoadjuvant chemoradiotherapy. Clin Cancer Res 2006; 12:97-106.

34. Wong CY, Schmidt J, Bong JS, et al. Correlating metabolic and anatomic responses of primary lung cancers to radiotherapy by combined F-18 FDG PET-CT imaging. Radiat Oncol 2007; 2:18.

35. Paesmans M, Berghmans T, Dusart M, et al. Primary tumor standardized uptake value measured on fluorodeoxyglucose positron emission tomography is of prognostic value for survival in non-small cell lung cancer: update of a systematic review and meta-analysis by the European Lung Cancer Working Party for the International Association for the Study of Lung Cancer Staging Project. J Thorac Oncol 2010; 5:612-9.

36. MacManus MP, Hicks RJ, Matthews JP, et al. Positron emission tomography is superior to computed tomography scanning for response-assessment after radical radiotherapy or chemoradiotherapy in patients with non-small-cell lung cancer. J Clin Oncol 2003; 21:1285-92. 
37. Cheung P, Faria S, Ahmed S, et al. A phase II study of accelerated hypofractionated 3-dimensional conformal radiation therapy for inoperable T1-3 N0 M0 non-small cell lung cancer: NCIC CTG BR.25. Int J Radiat Oncol Biol Phys 2012; 84:S47-8.

38. van Baardwijk A, Bosmans G, Dekker A, et al. Time trends in the maximal uptake of FDG on PET scan during thoracic radiotherapy: a prospective study in locally advanced non-small cell lung cancer (NSCLC) patients. Radiother Oncol 2007; 82: $145-52$.

39. Zhang HQ, Yu JM, Meng X, et al. Prognostic value of serial [18F]fluorodeoxyglucose PET-CT uptake in stage III patients with non-small cell lung cancer treated by concurrent chemoradiotherapy. Eur J Radiol 2011; 77:92-6.

40. Kong FM, Frey KA, Quint LE, et al. A pilot study of $[18 \mathrm{~F}]$ fluorodeoxyglucose positron emission tomography scans during and after radiation-based therapy in patients with non small-cell lung cancer. J Clin Oncol 2007; 25: 3116-23.
41. Aerts HJ, Bosmans G, van Baardwijk AA, et al. Stability of 18F-deoxyglucose uptake locations within tumor during radiotherapy for NSCLC: a prospective study. Int I Radiat Oncol Biol Phys 2008; 71:1402-7.

42. Wiegman EM, Pruim J, Ubbels JF, et al. 18F-FDG PET during stereotactic body radiotherapy for stage I lung tumours cannot predict outcome: a pilot study. Eur J Nucl Med Mol Imaging 2011; 38:1059-63.

43. Machtay M, Duan F, Siegel BA, et al. Prediction of survival by $[18 \mathrm{~F}]$ fluorodeoxyglucose positron emission tomography in patients with locally advanced non-small-cell lung cancer undergoing definitive chemoradiation therapy: results of the ACRIN 6668/RTOG 0235 trial. J Clin Oncol 2013; 31:3823-30.

44. Feng M, Kong FM, Gross M, et al. Using fluorodeoxyglucose positron emission tomography to assess tumor volume during radiotherapy for non-small-cell lung cancer and its potential impact on adaptive dose escalation and normal tissue sparing. Int J Radiat Oncol Biol Phys 2009; 73:1228-34. 